\title{
Metazoan parasite communities of two deep-sea elasmobranchs: the southern lanternshark, Etmopterus granulosus, and the largenose catshark, Apristurus nasutus, in the Southeastern Pacific Ocean
}

\author{
Juan F. Espínola-Novelo ${ }^{1}$, Rubén Escribano ${ }^{2,3}$, and Marcelo E. Oliva ${ }^{3,4, *}$ \\ ${ }^{1}$ Programa de Doctorado en Ciencias Aplicadas, mención Sistemas Marinos Costeros, Universidad de Antofagasta, P.O. Box 179, \\ Antofagasta, Chile \\ 2 Departamento Oceanografía, Universidad de Concepción, P.O. Box 160-C, Concepción, Chile \\ ${ }^{3}$ Millennium Institute of Oceanography (IMO), Universidad de Concepción, P.O. Box 160-C, Concepción, Chile \\ ${ }^{4}$ Instituto de Ciencias Naturales Alexander von Humboldt, Universidad de Antofagasta, P.O. Box 170, Antofagasta, Chile
}

Received 3 April 2018, Accepted 29 October 2018, Published online 20 November 2018

\begin{abstract}
Two deep-sea shark species were obtained as by-catch of the local fishery of the Patagonian toothfish, Dissostichus eleginoides, at depths ranging from 1000 to $2200 \mathrm{~m}$ off central and northern Chile. A total of 19 parasite taxa were found in 133 specimens of the southern lanternshark, Etmopterus granulosus, $(n=120)$ and largenose catshark, Apristurus nasutus, $(n=13)$. Fourteen taxa (four Monogenea, one Digenea, four Cestoda, one Nematoda, two Copepoda, one Annelida and one Thecostraca) were found in E. granulosus, whereas five taxa (one Monogenea, two Cestoda and two Nematoda) were found in A. nasutus. Representatives of Cestoda showed higher values of prevalence and a greater intensity of infection; this pattern is consistent with reports for elasmobranchs, but the monogenean richness was higher than that previously reported for related deep-sea sharks. Regarding E. granulosus, a positive and significant correlation between host length and abundance was found for six (four ectoparasites, one mesoparasite, and one endoparasite) of the 14 taxa recorded, but prevalence was significantly correlated with host length only for the monogenean Asthenocotyle sp. Although the sample size for A. nasutus was limited, we compared richness, abundance, diversity and evenness at the infracommunity and component community levels. All of these variables were higher for E. granulosus, but at the infracommunity level, abundance was higher for A. nasutus. All the parasite taxa (except two) represent new host and geographical records.
\end{abstract}

Key words: Deep-sea sharks, metazoan parasites, biodiversity, Southeastern Pacific Ocean.

Résumé - Communautés des parasites métazoaires de deux élasmobranches de mer profonde, Etmopterus granulosus et Apristurus nasutus, dans le sud-est de l'océan Pacifique. Deux espèces de requins des grands fonds ont été obtenues comme prises accessoires de la pêcherie locale de Légine australe, Dissostichus eleginoides, à des profondeurs allant de 1000 à $2200 \mathrm{~m}$, au large du centre et du nord du Chili. Au total, 19 taxons de parasites ont été trouvés dans 133 spécimens du Sagre long nez Etmopterus granulosus $(n=120)$ et du Holbiche cyrano Apristurus nasutus $(n=13)$. Quatorze taxons (quatre Monogenea, un Digenea, quatre Cestoda, un Nematoda, deux Copepoda, un Annelida et un Thecostraca) ont été trouvés chez E. granulosus, tandis que cinq taxons (un Monogenea, deux Cestoda et deux Nematoda) ont été trouvés chez A. nasutus. Les représentants des Cestoda ont montré des valeurs de prévalence plus élevées et une plus grande intensité d'infection; ce schéma concorde bien avec ce qui a été rapporté pour les élasmobranches, mais la richesse des Monogenea était supérieure à celle précédemment rapportée pour les requins de profondeur apparentés. En ce qui concerne E. granulosus, une corrélation positive et significative entre la longueur de l'hôte et l'abondance a été constatée pour six des 14 taxons répertoriés (quatre ectoparasites, un mésoparasite et un endoparasite), mais la prévalence était significativement corrélée à la longueur de l'hôte uniquement pour le Monogène Asthenocotyle sp. Bien que la taille de l'échantillon d'A. nasutus soit limitée, nous avons comparé la richesse, l'abondance, la diversité et la régularité aux niveaux infracommunautaire et communautaire. Toutes ces variables étaient plus élevées pour E. granulosus, mais au niveau infracommunautaire, l'abondance était supérieure pour $A$. nasutus. Tous les taxons parasites, à l'exception de deux, représentent de nouvelles mentions géographiques et de nouveaux hôtes.

*Corresponding author: marcelo.oliva@uantof.cl

This is an Open Access article distributed under the terms of the Creative Commons Attribution License (http://creativecommons.org/licenses/by/4.0), which permits unrestricted use, distribution, and reproduction in any medium, provided the original work is properly cited. 


\section{Introduction}

The deep-sea ( $>200 \mathrm{~m}$ depth) is the habitat for $10-15 \%$ of the global ichthyofauna [29, 30]. Despite its limited primary production, important marine resources inhabit this environment [3]; consequently, as coastal fisheries have collapsed, the deep-sea region may offer new resources [41]. Accordingly, we need not only clear and adequate knowledge of these potential new resources but also a sound understanding of the biodiversity of these ecosystems [3, 29]. Parasites are an important component of any ecosystem, not only because of the number of species (which is higher than the number of free-living species) but also because of the role they play in the trophic web $[16,31]$. Deep-sea fish parasites have often been neglected, although they are an essential part of marine biodiversity [29]. Moreover, through the study of parasites, it is possible to obtain information about their hosts, such as their diet, migratory movements and population structures, and the biodiversity and human impacts in the ecosystems where they coexist [34, $56,59]$. Consequently, parasites can provide crucial clues about the features of the ecosystems inhabited by their hosts.

Knowledge of the parasite fauna harboured by deep-sea fishes is available for less than $10 \%$ of the ichthyofauna that inhabit these ecosystems [29, 30]. This is due to the economic and logistical difficulties in accessing the hosts that inhabit these ecosystems in comparison with fishes of commercial importance or those that inhabit coastal areas [30]; an alternative is to study hosts from the by-catch of deep-sea commercial species. Knowledge regarding the parasites of sharks is scarce [9], and it is even scarcer for the species that inhabit the deepsea. Of the 509 species of sharks known to date, no fewer than 250 are considered deep-sea species [58]. Parasitological studies of species that inhabit these ecosystems focus on the taxonomic description of some species or new host records, but few include the analysis of the parasite community [14, 15, 25, 28]. For the Southeastern Pacific Ocean (SEPO herein and after), the same scenario is evident. Of the 68 shark species that have been reported, at least 33 are considered deep-sea sharks [17]. Only five studies have been carried out on the parasites of deep-sea sharks for the SEPO [11, 23, 32, 53, 54]. These papers focus on the taxonomic description of some parasite species or the presence of a parasite in a host; so far, no study has been conducted to address the metazoan parasite communities of deep-sea sharks for the SEPO.

Etmopterus granulosus (Günther, 1880), the southern lanternshark, and Apristurus nasutus De Buen, 1959, the longnose catshark, are two species inhabiting the deep-sea in the SEPO; E. granulosus shows a wide distribution in the Southern Ocean, including the southern Indian, southern Pacific and southwestern Atlantic Oceans, whereas A. nasutus is found from the Gulf of Panama to central Chile [17]. Both species are caught as by-catch in the local fishery of the Patagonian toothfish, Dissostichus eleginoides Smitt, 1898, in central and northern Chile as well as by-catch of the orange roughy, Hoplostethus atlanticus Collett 1889, in the Juan Fernandez Archipelago. Our goal is to report, for the first time, the composition of the metazoan parasite communities of two deep-sea sharks from the SEPO, as well as to quantitatively describe the characteristics of their parasite community.

\section{Materials and methods}

A total of 133 specimens (E. granulosus $=120$, A. nasutus $^{2} 13$ ) of deep-sea sharks were obtained during 2015-2017 (see Supplementary material) from the by-catch of the local fishery of the Patagonian toothfish (D. eleginoides) along the northern $\left(22^{\circ} 16^{\prime} \mathrm{S} 70^{\circ} 38^{\prime} \mathrm{W}-23^{\circ} 26^{\prime} \mathrm{S} 70^{\circ} 43^{\prime} \mathrm{W}\right)$ and central $\left(35^{\circ} 5^{\prime} \mathrm{S}-72^{\circ} 53^{\prime} \mathrm{W}\right)$ Chilean coasts at depths ranging from 1000 to $2200 \mathrm{~m}$ (Fig. 1) using a deep-sea longline. The sharks were captured, stored in bags and immediately frozen $\left(-18{ }^{\circ} \mathrm{C}\right)$ on board and transported to the laboratory for parasitological analyses.

After thawing, the sharks were measured (total length to the nearest $1.0 \mathrm{~cm}$ ), dissected and examined for metazoan parasites (both ectoparasites and endoparasites). Parasites were recorded by species and abundance for each shark, fixed in AFA (alcohol: formalin: acetic acid), and then preserved in 70\% alcohol. Nematoda were cleared with Amann lactophenol. Digenea, Monogenea and Cestoda were stained (acetic carmine) and cleared with clove oil (Sigma-Aldrich) and then mounted in Eukitt medium (O. Kindler GmbH, Germany) [42]. Copepoda and Thecostraca were stored in ethanol (70\%) and dissected for taxonomic purposes. Parasites were identified to the lowest taxonomic level possible. The prevalence and mean intensity of infection were calculated [8].

The quantitative analysis was carried out at the infracommunity and community component levels. For the infracommunity level, we calculated the richness (number of parasite species per examined host), abundance (number of parasite individuals per examined host) and diversity (Brillouin index) using PRIMER v6, parasite species accumulation curves were constructed using the "vegan" package in R freeware [44]. The Berger-Parker dominance index was calculated as the number of individuals of the most abundant parasite species divided by the total number of parasites in a given fish host as indicated by Dallarés et al. [14]. The descriptors Shannon, Simpson and Inverse Simpson diversity index were calculated at the community component level using the "vegan" package in R freeware. Because the sample size for A. nasutus was small, the characteristics were described but not compared, as $38 \%$ of the specimens of $A$. nasutus were not parasitized.

Potential relationships between host body length and richness (number of species), abundance (total number of individuals) and prevalence (previous angular transformation of prevalence data) were explored using the Spearman correlation coefficient only for E. granulosus. The potential relationship between length and abundance was explored in the same manner. Because of the small sample size of $A$. nasutus, the quantitative analysis of its parasite fauna was not performed. The statistical analyses were performed with the Minitab 17 statistical software program and Primer v6 [1].

\section{Results}

A total of 120 specimens of E. granulosus and 13 specimens of $A$. nasutus were obtained from nine fishing events, two from the central and seven from the northern fishing zone. Because the sample size was small for some periods, 


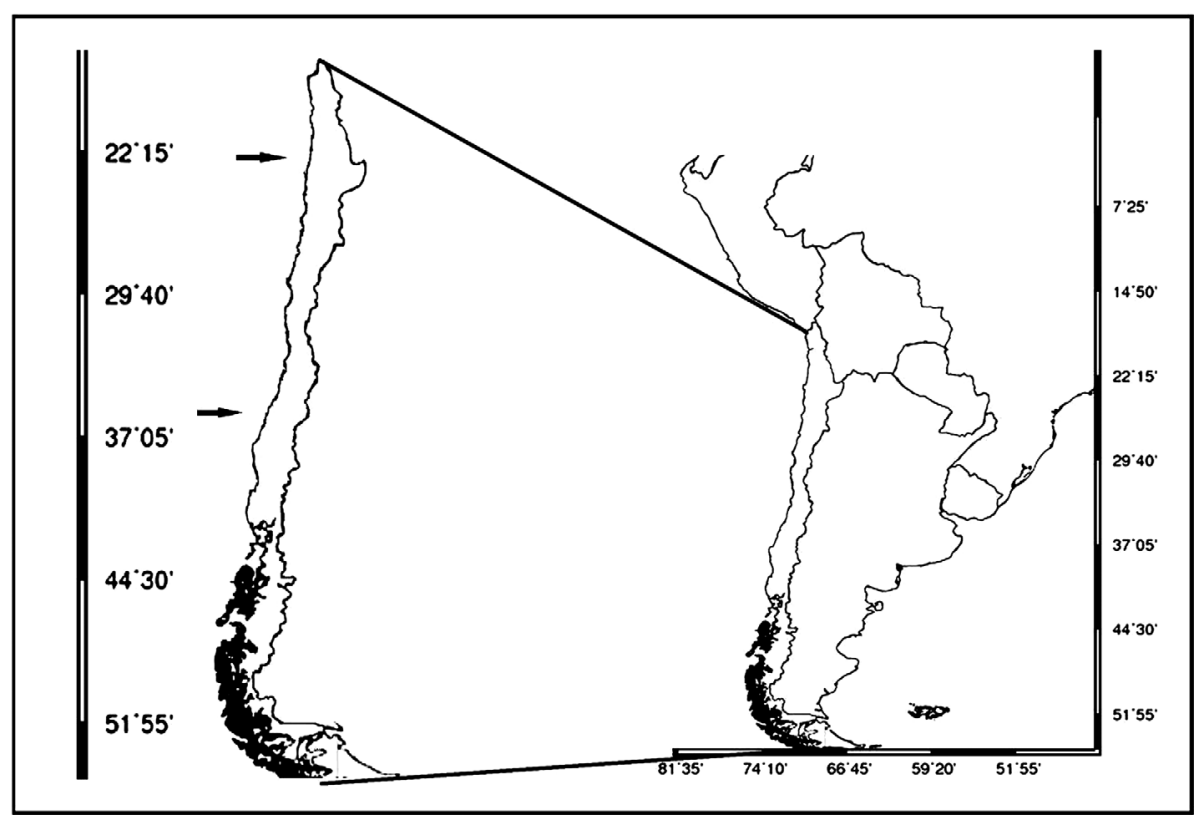

Figure 1. Approximate position of localities where samples of deep sea sharks were caught. Arrows indicate the approximate position of localities where samples of deep sea sharks were caught.

seasonality was not analysed (Supplementary material). The sharks were obtained with the same fishing gear.

The total length for E. granulosus ranged from 24 to $96 \mathrm{~cm}$ $(M=46.1 \pm 8.6)$, whereas the length range for $A$. nasutus was $52.5-84 \mathrm{~cm}(M=65.1 \pm 8.4)$. ANOVA showed that the mean length of the hosts differed between localities, but marginally $\left(F_{1,118}=3.98, p=0.048\right)$. Because the correlations between host length and total richness and total abundance were significant for both localities, the samples from northern and central sites were pooled.

A total of 277 parasite specimens belonging to 19 parasite taxa were obtained for the two hosts. Higher richness was found in E. granulosus, with 14 taxa: six endoparasites (one Digenea, four Cestoda and one Nematoda), seven ectoparasites (four Monogenea, two Copepoda, and one Annelida) and one mesoparasite (Thecostraca). Five taxa were found from $A$. nasutus; four were endoparasites (two Cestoda and two Nematoda) and one ectoparasite (Monogenea).

Four taxa of Monogenea were found in E. granulosus but with low values of prevalence and mean intensity. The monogenean Microbothrium sp. was found in A. nasutus, showing the highest mean intensity observed in this study (Table 1).

Two members of Copepoda were found: Lernaeopodidae gen sp. and Neoalbionella sp. The latter taxon showed the highest prevalence in E. granulosus. One annelid was found parasitizing E. granulosus; this leech was the least common taxon, with a single individual in the whole sample.

In both host species, Cestoda was well represented in terms of the number of taxa and individuals. In E. granulosus, three of four Cestoda were found in the adult stage, including Plesiorhynchus sp., which had the highest prevalence and mean intensity (Table 1), and one at the larval stage (Hepatoxylon sp.). The larval Hepatoxylon sp. was common for both sharks, showing the highest prevalence in A. nasutus. Of the three taxa of Nematoda, E. granulosus harboured one unidentified species of Anisakis, while A. nasutus harboured Anisakis sp. and Mooleptus rabuka. The digenean Otodistomum sp. was found only in E. granulosus, with low prevalence and abundance.

Individuals of the cosmopolitan mesoparasite Anelasma squalicola (Thecostraca) were found in both the dorsal and caudal fins, eyes and mouth of E. granulosus.

The sample size of E. granulosus allowed us to evaluate certain quantitative characteristics of the infection at the infracommunity and component levels (Table 2). Parasite species accumulative curves are shows in Figure 2, for both host species. The expected number of species ranged from 14.8 (Bootstrap) to 17.9 (Jackknife2) for $E$. granulosus and 5.9 (Bootstrap) and 6.9 (Jackknife2) for A. nasutus (Table 2).

The richness, abundance and diversity were positively and significantly correlated with host length $(r=0.408$, $p<0.001, d f=118 ; r=0.436, p<0.001, d f=118 ; \quad r=$ $0.41, p<0.001, d f=65$, respectively). The abundance of some taxa (Asthenocotyle sp. $(r=0.322, p<0.001, d f=118)$, Monocotylidae gen. sp. $(r=0.228, p=0.012, d f=118)$, Plesiorhynchus sp. $(r=0.286, \quad p=0.002, \quad d f=118)$, Lernaeopodidae gen. sp. $(r=0.184, p=0.044, d f=118)$, Neoalbionella sp. $(r=0.237, p=0.009, d f=118)$, and $A$. squalicola $(r=0.293, p=0.001, d f=118)$ ) was positively and significantly correlated with host length. The prevalence of infection was significantly correlated with host length only for Asthenocotyle sp. $(r=0.94, p<0.001, d f=3)$.

\section{Discussion}

Hosts were obtained using the same fishing gear (deep-sea longline), on the same date and with the same fishing effort. Accordingly, the differences in the number of hosts obtained suggest differences in the relative abundance of each species. A similar result (higher abundance of E. granulosus than 
Table 1. Prevalence $(\mathrm{P})$ and mean intensity \pm standard deviation $(M I \pm S D)$ of infection of metazoan parasites found in two species of deep-sea sharks from the SEPO.

\begin{tabular}{|c|c|c|c|c|c|c|}
\hline \multirow[b]{2}{*}{ Parasite species } & \multirow[b]{2}{*}{ Stage } & \multirow[b]{2}{*}{ Habitat } & \multicolumn{2}{|c|}{ E. granulosus } & \multicolumn{2}{|c|}{ A. nasutus } \\
\hline & & & $\mathrm{P}$ & $M I \pm S D$ & $\mathrm{P}$ & $M I \pm S D$ \\
\hline \multicolumn{7}{|l|}{ Monogenea } \\
\hline Squalonchocotyle aff. spinacis & A & G & 5.1 & $1.2 \pm 0.4$ & - & - \\
\hline Asthenocotyle sp. & $\mathrm{A}$ & $\mathrm{S}$ & 9.3 & $1.5 \pm 0.5$ & - & - \\
\hline Microbothrium sp. & $\mathrm{A}$ & $\mathrm{S}$ & - & - & 15.3 & $24.0 \pm 3.0$ \\
\hline Calicotyle sp. & $\mathrm{A}$ & $\mathrm{S}$ & 1 & $2 \pm 0$ & - & - \\
\hline $\begin{array}{l}\text { Monocotylidae gen. sp. } \\
\text { Digenea }\end{array}$ & $\mathrm{A}$ & $\mathrm{S}$ & 3.4 & $1.5 \pm 0.6$ & - & - \\
\hline $\begin{array}{l}\text { Otodistomum } \mathrm{sp} \\
\text { Cestoda }\end{array}$ & A & I & 5.1 & $1.2 \pm 0.4$ & - & - \\
\hline Aporhynchus sp. & A & SV & 5.9 & $1.2 \pm 0.4$ & - & - \\
\hline Plesiorhynchus sp. & A & SV & 17.8 & $5.6 \pm 4.9$ & - & - \\
\hline Hepatoxylon sp. & $\mathrm{L}$ & $\mathrm{Me}$ & 5.1 & $1.3 \pm 0.5$ & 53.8 & $1.7 \pm 1.4$ \\
\hline Trypanorhyncha gen. sp. & $\mathrm{L}$ & $\mathrm{Me}$ & - & - & 7.7 & $1.0 \pm 0$ \\
\hline $\begin{array}{l}\text { Cestoda unidentified } \\
\text { Nematoda }\end{array}$ & & SV & 12.7 & $2.2 \pm 2.1$ & - & - \\
\hline Anisakis sp. & $\mathrm{L}$ & $\mathrm{I}, \mathrm{M}$ & 13.3 & $1.0 \pm 0$ & 15.4 & $1.5 \pm 0.5$ \\
\hline $\begin{array}{l}\text { Mooleptus rabuka } \\
\text { Copepoda }\end{array}$ & A & I & - & - & 7.7 & $1.0 \pm 0$ \\
\hline Lernaeopodidae gen sp. & $\mathrm{A}$ & $\mathrm{B}$ & 2.5 & $1.0 \pm 0$ & - & - \\
\hline $\begin{array}{l}\text { Neoalbionella } \mathrm{sp} . \\
\text { Thecostraca }\end{array}$ & $\mathrm{A}$ & $\mathrm{F}$ & 12.7 & $1.1 \pm 0.5$ & - & - \\
\hline $\begin{array}{l}\text { Anelasma squalicola } \\
\text { Annelida }\end{array}$ & $\mathrm{A}$ & S, E, F, Mo & 13.6 & $2.4 \pm 0$ & - & - \\
\hline Piscicolidae gen sp. & $\mathrm{A}$ & $\mathrm{S}$ & 0.8 & $1.0 \pm 0$ & - & - \\
\hline
\end{tabular}

$\mathrm{A}=$ adult, $\mathrm{E}=$ eyes, $\mathrm{F}=$ fins, $\mathrm{G}=$ Gills, $\mathrm{I}=$ intestine, $\mathrm{L}=$ Larval stage, $\mathrm{Me}=$ mesenteries, $\mathrm{Mo}=$ Mouth, $\mathrm{SV}=\mathrm{Spiral}$ valve, $\mathrm{S}=\mathrm{Skin}$.

Table 2. Quantitative characteristics of the metazoan parasites in two deep-sea sharks at component and infracommunity level.

\begin{tabular}{lcc}
\hline & E. granulosus & A. nasutus \\
\hline $\begin{array}{l}\text { Component community } \\
\text { Observed richness }\end{array}$ & 14 & \\
Expected richness & & 5 \\
$\quad$ Chao & $14.9(S E=2.3)$ & $5.9(S E=1.7)$ \\
Jackknife 1 & $15.9(S E=1.4)$ & $6.8(S E=1.3)$ \\
Jackknife 2 & 17.9 & 6.9 \\
Bootstrap & $14.8(S E=0.7)$ & $5.9(0.7)$ \\
Abundance & 282 & 65 \\
Diversity & $1.9603(S D=0.064)$ & $0.806(S D=0.121)$ \\
Evenness & 0.738 & 0.501 \\
Infracommunity & & \\
Richness & $1.06( \pm 1.27)$ & $0.92( \pm 0.86)$ \\
Abundance & $2.35(S D=3.71)$ & $5(S D=9.30)$ \\
Diversity & $0.26(S D=0.32)$ & $0.13(S D=0.15)$ \\
Evenness & $0.42(S D=0.46)$ & $0.32(S D=0.43)$ \\
\hline
\end{tabular}

$S E=$ standard error, $S D=$ standard deviation.

A. nasutus) was reported for the by-catch of the orange roughy, Hoplostethus atlanticus, in the SEPO [39].

This is the first study reporting the composition of the metazoan parasite community in the deep-sea sharks E. granulosus and A. nasutus. Most parasitological studies on deep-sea sharks have focused on the taxonomic description of a given parasite species or recorded the presence of parasite species in a given host, with few studies describing the species composition at the community level [13-15, 25, 28]. Previous studies regarding the parasites of $E$. granulosus and $A$. nasutus consist of taxonomic records.

For E. granulosus, there are six taxonomic records: O. plunketi from New Zealand [7, 22], G. squali from Chile, A. tasmaniensis and P. etmopteri from Australia [5, 11], Neoalbionella sp. from Juan Fernandez Archipelago, Chile [53], and A. squalicola from New Zealand [27, 60]. Accordingly, Squalonchocotyle aff. spinacis, Asthenocotyle sp., Calicotyle sp., Monocotylidae gen. sp., Otodistomum sp., Aporhynchus sp., Plesiorhynchus sp., Hepatoxylon sp., Anisakis sp., Lernaeopodidae gen sp. and Piscicolidae gen. sp. represent new records for E. granulosus. The Neoalbionella sp. now found could be the same taxon reported by Rodríguez et al. [53]. Unfortunately, males were not found, and they are necessary for the correct identification of the species; therefore, Neoalbionella sp. and A. squalicola represent new locality records. For $A$. nasutus, the only previous record is the nematode $M$. rabuka [54]; thus, the four remaining taxa (Microbothrium sp. Hepatoxylon sp., Trypanorhyncha gen. sp. and Anisakis sp.) represent new records for A. nasutus.

The most common taxon of parasites in elasmobranchs is Platyhelminthes, followed by Arthropoda, Nematoda, Annelida and Acanthocephala [9]. Our data align well with this pattern for both host species analysed. These authors also suggest that 

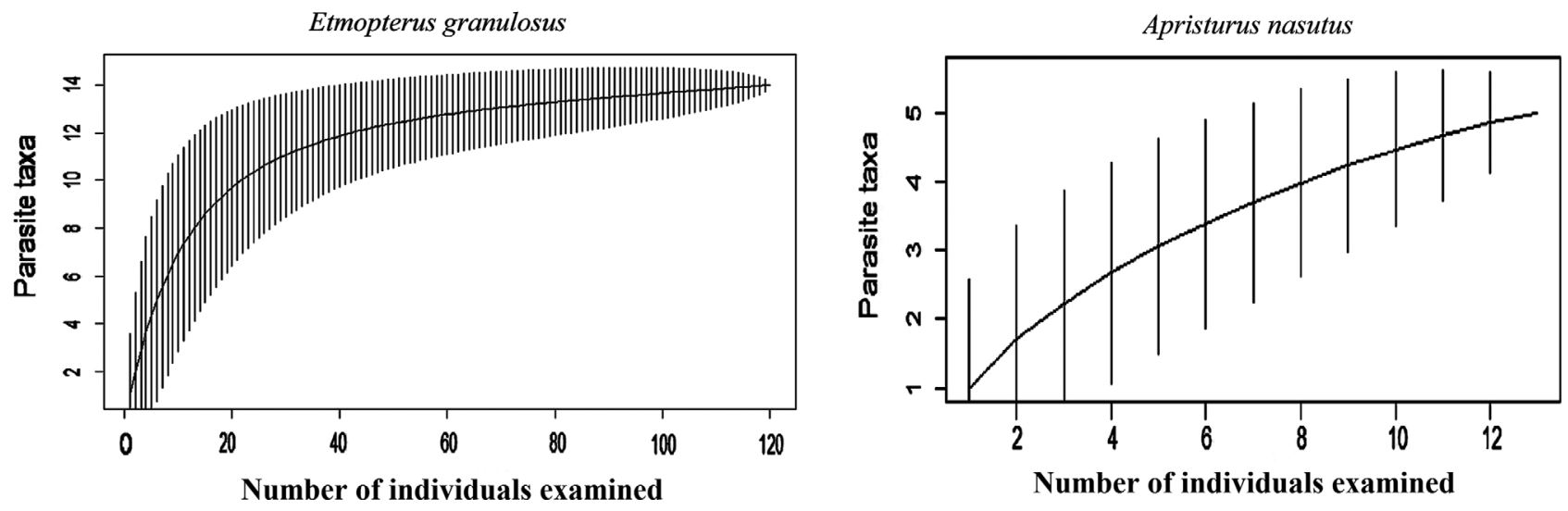

Figure 2. Species accumulation curves for both host species studied.

the richest group in the Platyhelminthes is Cestoda, followed by Monogenea and Digenea.

Parasitological studies are available for seven species of Etmopterus; of these studies, only three include quantitative data, but only for $E$. spinax $[14,25,28]$. Our data suggest that E. granulosus shows the highest richness among members of this genus (14 taxa) based on the published quantitative data (Supplementary material), but the integration of published taxonomic information (14 articles from seven localities) indicates the presence of 23 species, with Cestoda being the best represented group (14 taxa) (Supplementary material). With regard to Monogenea, E. granulosus showed the highest richness, with four taxa. Monogeneans have been reported only for two members of the Etmopterus genus. In regard to A. nasutus, the highest richness corresponds to Cestoda (two taxa) and Nematoda (two taxa). Among ectoparasites of elasmobranchs, Copepoda is the most diverse group, followed by Monogenea [9]. Our data show the opposite pattern: Monogenea was the richest group in E. granulosus. Copepoda was represented by two taxa, but specimens of Neoalbionella sp. seem in fact to be members of two species (Castro pers. comm.).

A different picture is evident for members of Apristurus. Taxonomic studies are available for seven species. The integration of taxonomic papers indicates richness ranging from one to two species rather than the five taxa found here. Any conclusion about richness in A. nasutus must be drawn with caution due to our small sample size and the absence of quantitative data for other members of the genus. However, the sample effort for both species captures the expected richness (Table 2).

Many factors have been proposed as drivers of the structure of parasitic communities, among others the diet of the host (generalist versus specialist predators), ontogenetic changes in the diet, prey availability (intermediate hosts) [50], as well as habitat, host behaviour (migratory versus sedentary, schooling versus non-schooling) and host density. Environmental factors, such as depth and water temperature, also influence community structure $[19,48,50]$. Recently [36], determinants of parasite species richness were evaluated, but no clear conclusions were obtained. Based on a meta-analysis, Kamiya et al. [26] suggest four potential universal predictors of richness, and three of them (host body length, geographical range size and population density) were adequate predictors, while latitude was not. Our results for E. granulosus are in partial accordance with those postulated, showing that a greater geographical range was related to higher parasitic richness. With respect to density, no data on host density are available, but because both species were obtained with the same fishing gear and sampling effort, this suggests that $E$. granulosus has a higher density than $A$. nasutus. However, when host length was evaluated, our results showed the opposite pattern to that expected according to the predictions of Kamiya et al. [26], as the mean length of $A$. nasutus was higher than that of E. granulosus. The positive and significant correlation found between host length and richness for E. granulosus suggests that for a given species, length is a suitable predictor of richness.

Six of the 14 taxa found in E. granulosus (Asthenocotyle sp., Monocotylidae gen. sp., Lernaeopodidae gen sp., Neoalbionella sp., A. squalicola and Plesiorhynchus sp.) showed a positive and significant correlation between abundance and host length (as a proxy for host age), suggesting cumulative infection with age or a colonisation rate that is higher than the mortality rate [47]. The prevalence of infection was significantly correlated with host length only for Asthenocotyle sp.

Despite the high diversity at the community component level for E. granulosus, at the infracommunity level, the richness and diversity showed low values that were similar to those reported for members of Etmopterus in other localities (Supplementary material).

The four taxa of monogenean recorded from E. granulosus represent unusually high richness for this group in deep-sea sharks. Just two species were recorded in 37 specimens of E. spinax [28], and Isber et al. [25] found only one species in a sample of 59 specimens. Moreover, Klimpel et al. [30] listed the parasite fauna of 30 shark species; of those, just three (Hexanchus griseus, Etmopterus spinax and Dipturus oxyrinchus) harbour two species of Monogenea, and the remaining 27 species harbour one or no species. A similar pattern was suggested $[42,43]$ in deep-sea teleosts in the SEPO. Although a small proportion of deep-sea teleosts and sharks have been studied, our results and those of Nacari et al. [42] 
could be evidence that higher Monogenea richness in the SEPO is driven by environmental conditions.

Monogeneans found in E. granulosus belong to Hexabothriidae, Microbothriidae and Monocotylidae. Members of the Hexabothriidae and Monocotylidae are parasites of elasmobranchs and holocephalans [6, 12], whereas Microbothriidae are parasites of elasmobranchs only [57].

Two copepods were found in E. granulosus. Unfortunately, all the individuals found were females, and it was thus not possible to identify them to lower taxonomic levels. Both taxa belong to the Lernaeopodidae family. Members of the Neoalbionella genus are parasites of sharks of the Etmopteridae and Pentanchidae families [4, 24, 35, 53, 55].

The monotypic genus Anelasma is the only barnacle parasite of vertebrates [51]. This crustacean has been considered to be an ectoparasite [32], but it can be considered to be a mesoparasite, since it is partially embedded and partially exposed to the environment [9, 60]. A. squalicola (and also Anelasma sp.) has been reported only in sharks belonging to Etmopteridae [30], suggesting high host specificity.

Both presence of the adult and larval stages of endoparasites indicates that the studied sharks may function as final as well as intermediate hosts. Although the predators of E. granulosus and A. nasutus are unknown, small sharks are an important component of the diet of larger sharks; for example, small sharks such as Galeus melastomus and Etmopterus spinax are preyed upon by Dalatias licha [38].

Larval cestodes (Hepatoxylon sp. and Trypanorhyncha gen. sp.) can reach the adult stage in shark predators. Members of the Hepatoxylon genus (found in both hosts) are common parasites in teleosts (larval forms) and elasmobranchs (larval and adults) and have been reported from more than 40 species of sharks (see [49]). They have not been reported in sharks of the Etmopterus and Apristurus genera. The low prevalence and mean intensity in E. granulosus suggest accidental infection, but the high prevalence and mean intensity in A. nasutus suggests that this species is an intermediate host for this cestode. The lack of dietary data for these sharks prevents any conclusion about the life cycle of this worm. Merluccids and macrourids are part of the diet of E. granulosus in New Zealand [17]. In the SEPO, the south Pacific hake (Merluccius gayi), the Patagonian grenadier (Macruronus magellanicus (Merlucciidae)) and the bigeye grenadier Macrourus holotrachys (Macrouridae) are parasitized by larval Hepatoxylon trichiuri with high prevalence $(>60 \%$ in $M$. magellanicus) [42, 45, 46], suggesting that E. granulosus may be infected with this larval cestode by preying on these fish species.

Although the life cycles of adult cestodes found in E. granulosus (Aporhynchus sp. and Plesiorhynchus sp) are not known, the feeding habits of E. spinax, suggest that the calanoid Calanus finmarchicus may be the first intermediate host for Aporhynchus norvegicus, while Meganyctiphanes norvegica is the second intermediate host [28].

Aporhynchus sp. and Plesiorhynchus sp. present some degree of host specificity. Currently, the trypanorhynch genus Aporhynchus comprises four species that infect three etmopterids: A. norvegicus and A. menezesi in E. spinax, A. tasmaniensis in E. granulosus (= baxteri) and A. pickeringae in E. pusillus; [40], but Aporhynchus sp. was detected in Deania profundorum, a member of Centrophoridae [10]. Plesiorhynchus includes three species; all of them have been found in deep-sea sharks, P. campbelli was found in Apristurus sp. [5] and in the etmopterids: E. princeps was infected with $P$. brayi and E. lucifer, and E. granulosus with P. etmopteri [5, 10].

Of the four nematodes found, two belong to the Anisakis genus. Adult Anisakis parasitize marine mammals, and larval anisakids are rare in sharks [28]. As stated by Henderson et al. [20], they must be considered accidental parasites; however, their presence has been reported in Prionace glauca [20], Squalus acanthias [21], Centrophorus squamosus [13], and E. spinax [28], as well as in the blackmouth catshark (Galeus melastomus (Pentanchidae)) [14, 15].

In $A$. nasutus, two nematodes were found: Anisakis sp. and Mooleptus rabuka. The latter species has been found in Apristurus fedorovi and Apristurus japonicus [2, 37]. Larval forms, presumed members of this nematode genus, have been found in the brain of the deep-water teleost Cyclothone atraria [33].

Despite its low prevalence and abundance, the presence of the digenean Otodistomum sp. cannot be the result of accidental infections; members of this genus have elasmobranchs as main hosts [9], and they have been reported in E. granulosus (and E. baxteri) [7, 22] and other members of Etmopterus, such as E. princeps [18] and E. spinax [25]. Their low abundance could be related to the low availability of intermediate hosts, such as some teleost fishes, the second intermediate host for this digenean [52].

\section{Conclusions}

The present study analysed for the first time the composition of the metazoan parasite communities in two deep-sea sharks from the SEPO. The richness found in E. granulosus is the highest among members of the Etmopterus genus. Twelve of the 14 parasite taxa found represent new records for this species, whereas for A. nasutus, four of five parasites represent new host records. The species composition for both host species analysed showed a pattern similar to that reported for other elasmobranchs, i.e., higher richness of Platyhelminthes, mainly Cestoda and also Monogenea. The diversity of Monogenea parasitizing E. granulosus is higher than that found in previous reports for elasmobranchs, and a pattern similar to that described for deep-sea teleosts in the SEPO is evident.

The presence of the cosmopolitan A. squalicola in E. granulosus suggests high connectivity in the deep sea, at least in the Southern Hemisphere.

\section{Supplementary Material}

Supplementary material is available at https://www. parasite-journal.org/10.1051/parasite/2018054/olm

Acknowledgements. We appreciate the kind support of the crew of the fishing boat "Doña Bella" and its Captain Mr. Dani Manzo, as well as Raul Castro and Luis Nacari for their help in the taxonomic identification of some parasites, and Carolina Burgos, Marie-Charlotte Cheutin and Florence Droguet for assistance in 
the laboratory. Partially funded by FONDECYT (National Fund for Science and Technology) 1140173. The Millennium Institute of Oceanography (IMO), IC120019 also provided support. The authors declare that there is no conflict of interest.

\section{References}

1. Anderson MJ, Gorley RN, Clarke KR. 2008. PERMANOVA+ for PRIMER: Guide to software and statistical methods. Plymouth, UK: PRIMER-E.

2. Ando T, Nagasawa K, Nakaya K. 2002. Stomach contents and nematode infection of two deep-water catsharks, Apristurus fedorovi and A. japonicus, from Northern Japan. Bulletin of Fisheries Sciences, Hokkaido University, 53, 41-43.

3. Armstrong CW, Foley NS, Tinch R, van den Hove S. 2012. Services from the deep: Steps towards valuation of deep-sea goods and services. Ecosystem Services, 2, 2-13.

4. Benz GW. 1991. Description of some larval stages and augmented description of adult stages of Albionella etmopteri (Copepoda: Lernaeopodidae), a parasite of deep-water lanternsharks 0(Etmopterus: Squalidae). Journal of Parasitology, 77, 666-674.

5. Beveridge I. 1990. Revision of the family Gilquiniidae Dollfus (Cestoda, Trypanorhyncha) from elasmobranch fishes. Australian Journal of Zoology, 37, 481-520.

6. Boeger WA, Kritsky DC. 1989. Phylogeny, coevolution, and revision of the Hexabothriidae Price, 1942 (Monogenea). International Journal of Parasitology, 19, 425-440.

7. Bray RA, Cribb TH. 2003. The digeneans of elasmobranches distribution and evolutionary significance, in Taxonomy, Ecology and Evolution of Metazoan Parasites. (Livre hommage á Louis Euzet). Tome I, Combes C, Jourdane J, Editors. Presses Universitaires de Perpignan: Perpignan France. p. 67-96.

8. Bush AO, Lafferty KD, Lotz JM, Shostak AW. 1997. Parasitology meets ecology on its own terms: Margolis et al. revisited. Journal of Parasitology, 83, 575-583.

9. Caira J, Healy C. 2004. Elasmobranchs as hosts of metazoan parasites, in Biology of Sharks and Their Relatives, Marine Biology, Carrier JC, Musick JA, Heithaus MR, Editors. CRC Press: Florida. p. 523-551.

10. Caira JN, Pickering M. 2013. Cestoda from deep-water squaliform sharks in the Azores. Deep-Sea Research Part II Topical Studies in Oceanography, 98, 170-177.

11. Carvajal J. 1974. Records of cestodes from Chilean sharks. Journal of Parasitology, 60, 29-34.

12. Chisholm LA, Hansknecht TJ, Whittington ID, Overstreet RM. 1997. A revision of the Calicotylinae Monticelli, 1903 (Monogenea: Monocotylidae). Systematic Parasitology, 38, 159-183.

13. Costa G, Chada T, Melo-Moreira E, Cavallero S, D'Amelio S. 2014. Endohelminth parasites of the leafscale gulper shark, Centrophorus squamosus (Bonnaterre, 1788) (Squaliformes: Centrophoridae) off Madeira Archipelago. Acta Parasitologica, 59, 316-322.

14. Dallarés S, Padrós F, Cartes JE, Soléb M, Carrassón M. 2017. The parasite community of the sharks Galeus melastomus, Etmopterus spinax and Centroscymnus coelolepis from the NW Mediterranean deep-sea in relation to feeding ecology and health condition of the host and environmental gradients and variables. Deep-Sea Research Part I Oceanographic Research Papers, 129, 41-58.

15. Dallarés S, Pérez-del-Olmo A, Montero FE, Carrasón M. 2017. Composition and seasonal dynamics of the parasite communities of Scyliorhinus canicula (L. 1758) and Galeus melastomus Rafinesque, 1810 (Elasmobranchii) from the NW
Mediterranean Sea in relation to host biology and ecological features. Hydrobiologia, 799, 275-291.

16. Dobson A, Lafferty KD, Kuris AM, Hechinger RF, Jetz W. 2008. Colloquium paper: homage to Linnaeus: how many parasites? How many hosts? Proceedings of the National Academy of Sciences of the United States of America, 105, Suppl, 1148211489.

17. Ebert DA. 2016. Deep-sea cartilaginous fishes of the southeastern Pacific Ocean, in: FAO. Species Catalogue for Fishery Purposes, FAO: Rome, Italy.

18. Gibson DI, Bray RA. 1977. The Azygiidae, Hirudinellidae, Ptychogonimidae, Sclerodistomidae and Syncoeliidae (Digenea) of fishes from the northeast Atlantic. Bulletin of the British Museum (Natural History) Zoology, 32, 167-245.

19. González MT, Oliva ME. 2006. Similarity and structure of the ectoparasite communities of rock fish species from the southern Chilean coast in a temporal scale. Parasitology, 133, 335-343.

20. Henderson AC, Flannery K, Dunne J. 2002. Parasites of the blue shark (Prionace glauca L.), in the North-East Atlantic Ocean. Journal of Natural History, 36, 1995-2004.

21. Henderson AC, Flannery K, Dunne J. 2002. An investigation into the metazoan parasites of the spiny dogfish (Squalus acanthias L.), off the west coast of Ireland. Journal of Natural History, 36, 1747-1760.

22. Hine PM, Jones JB, Diggles BK. 2000. A checklist of the parasites of New Zealand fishes, including previously unpublished records. NIWA Technical report 75, 95 p. Available at https://trove.nla.gov.au/version/44096003.

23. Ho JS, McKinney LE. 1981. A new species of Eudactylina (Copepoda, Eudactylinidae) parasitic on black shark from Chile. Proceedings of the Biological Society of Washington, 94, $745-752$.

24. Hogans WE, Marques F. 1994. Albionella kabatai Benz \& Izawa, 1993 (Copepoda: Lernaeopodidae) from Apristurus profundorum (Goode \& Bean, 1896) (Chondrichthyes: Scyliorhinidae) in the northwest Atlantic Ocean. Belgian Journal of Zoology, 124, 193-198.

25. Isbert W, Rodríguez-Cabello C, Frutos I, Preciado I, Montero FE, Pérez-del-Olmo A. 2015. Metazoan parasite communities and diet of the velvet belly lantern shark Etmopterus spinax (Squaliformes: Etmopteridae): a comparison of two deep-sea ecosystems. Journal of Fish Biology, 86, 687-706.

26. Kamiya T, O’Dwyer K, Nakagawa S, Poulin R. 2014. What determines species richness of parasitic organisms? A metaanalysis across animal, plant and fungal hosts. Biological reviews of the Cambridge Philosophical Society, 89, 123-134.

27. Kazachensko VN, Feshchenko NV. 2016. Discovery of Anelasma squalicola (Cirripedia, Anelasmatidae) at new host Etmopterus baxteri (Squaliformes, Etmopteridae), Far Eastern State Technical Fisheries University: UDC 591.69.7, 17-19 (in Russian). Vladivostok.

28. Klimpel S, Palm HW, Seehagen A. 2003. Metazoan parasites and food composition of juvenile Etmopterus spinax (L., 1758) (Dalatiidae, Squaliformes) from the Norwegian Deep. Parasitology Research, 89, 245-251.

29. Klimpel S, Palm HW, Busch MW, Kellermanns E, Rückert S. 2006. Fish parasites in the Arctic deep-sea: Poor diversity in pelagic fish species vs. heavy parasite load in a demersal fish. Deep-Sea Research Part I Oceanographic Research Papers, 53, 1167-1181.

30. Klimpel S, Busch MW, Kellermanns E, Kleinertz S, Palm HW. 2009. Metazoan deep-sea fish parasites. Germany, Heinrich Heine Universität.

31. Lafferty KD, Allesina S, Arim M, Briggs CJ, De Leo G, Dobson AP, Dunne JA, Johnson PTJ, Kuris AM, Marcogliese 
DJ, Martinez DN, Memmott J, Marquet PA, McLaughlin JP, Mordecai EA, Pascual M, Poulin R, Thieltges DW. 2008. Parasites in food webs: the ultimate missing links. Ecology Letters, 11, 533-546.

32. Long DJ, Waggoner BM. 1993. The ectoparasitic barnacle Anelasma (Cirripedia, Thoracica, Lepadomorpha) on the shark Centroscyllium nigrum (Chondrichthyes, Squalidae) from the Pacific sub-Antarctic. Systematic Parasitology, 26, 133-136.

33. Machida M, Ogawa K, Okiyama M. 1982. A new nematode (Spirurida, Physalopteridae) from frill shark of Japan. Bulletin of the National Museum of Nature and Science. Series A, Zoology, 8, 1-5.

34. Marcogliese DJ. 2005. Parasites of the superorganism: Are they indicators of ecosystem health? International Journal of Parasitology, 35, 705-716.

35. Moore ABM. 2001. Metazoan parasites of the lesser-spotted dogfish Scyliorhinus canicula and their potential as stock discrimination tools. Journal of the Marine Biological Association of the United Kingdom, 81, 1009-1013.

36. Morand S. 2015. (macro-) Evolutionary ecology of parasite diversity: From determinants of parasite species richness to host diversification. International Journal for Parasitology: Parasites and Wildlife, 4, 80-87.

37. Moravec F, Nagasawa K. 2000. Two remarkable nematodes from sharks in Japan. Journal of Natural History, 34, 1-13.

38. Navarro J, López L, Coll M, Barría C, Sáez-Liante R. 2014. Short-and long-term importance of small sharks in the diet of the rare deep-sea shark Dalatias licha. Marine Biology, 161, 1697-1707.

39. Niklitschek EJ, Cornejo-Donoso J, Oyarzún C, Hernández E, Toledo P. 2010. Developing seamount fishery produces localized reductions in abundance and changes in species composition of bycatch. Marine Ecology, 31, 168-182.

40. Noever C, Caira JN, Kuchta R, Desjardins L. 2010. Two new species of Aporhynchus (Cestoda: Trypanorhyncha) from deep water lanternsharks (Squaliformes: Etmopteridae) in the Azores, Portugal. Journal of Parasitology, 96, 1176-1184.

41. Norse EA, Brooke S, Cheung WWL, Clark MR, Ekeland I, Froese R, Gjerde KM, Haedrich RL, Heppell SS, Morato T, Morgank LE, Pauly D, Sumaila R, Watson R. 2012. Sustainability of deep-sea fisheries. Marine Policy, 36, 307-320.

42. Ñacari LA, Oliva ME. 2016. Metazoan parasites of deep-sea fishes from the South Eastern Pacific: Exploring the role of ecology and host phylogeny. Deep-Sea Research Part I Oceanographic Research Papers, 115, 123-130.

43. Nacari LA, Sepulveda FA, Escribano R, Oliva ME. 2018. Acanthocotyle gurgesiella $\mathrm{n}$. sp. (Monogenea: Acanthocotylidae) from the deep-sea skate Gurgesiella furvescens (Rajidae) in the south-eastern Pacific. Journal of Helminthology, 92, 223-227.

44. Oksanen J, Blanchet FG, Friendly M, Kindt R, Legendre P, McGlinn D, Minchin PR, O'Hara RB, Simpson GL, Solymos P, Stevens M, Henry H, Szoecs E, Wagner H. 2018. Vegan: Community ecology package, $\mathrm{R}$ package version $2.5-2$. Available at https://cran.r-project.org package=vegan (accessed 11 August 2018).

45. Oliva ME. 2001. Metazoan parasites of Macruronus magellanicus from southern Chile as Biological tags. Journal of Fish Biology, 58, 1617-1622.

46. Oliva ME, Ballón I. 2002. Parasites of the Chilean hake Merluccius gayi as a tool for stocks discrimination. Fisheries Research, 56, 313-320.
47. Oliva ME, Díaz M. 1988. Aspectos cuantitativos de la infección por Proctoeces humboldti (Trematoda: Fellodistomidae) en la lapa Fissurella crassa (Mollusca: Archaeogastropoda). Revista Chilena de Historia Natural, 61, 27-33.

48. Oliva ME, González M, Acuña E. 2004. Metazoan parasite fauna as a biological tag for the habitat of the flounder Hippoglossina macrops from northern Chile, in a depth gradient. Journal of Parasitology, 90, 1374-1377.

49. Pollerspöck J, Straube N. 2015. Bibliography database of living/fossil sharks, rays and chimaeras (Chondrichthyes: Elasmobranchii, Holocephali) Host-Parasites List/ Parasite-Hosts List-, www.shark-references.com, World Wide Web electronic publication, Version 04/2015 ISSN: 2195-6499

50. Poulin R. 1995. Phylogeny, ecology, and the richness of parasite communities in vertebrates. Ecological Monographs, $65,283-302$

51. Rees DJ, Noever C, Høeg JT, Ommundsen A, Glenner H. 2014. On the origin of a novel parasitic-feeding mode within suspension-feeding barnacles. Currents in Biology, 24, 1429-1434.

52. Rocka A. 2006. Helminths of Antarctic fishes: Life cycle biology, specificity and geographical distribution. Acta Parasitologica, 51, 26-35.

53. Rodríguez SM, George-Nascimento M, Luque JL. 2010. A parasitic copepod, Neoalbionella sp. (Lernaeopodidae), on the southern lanternshark Etmopterus granulosus (Etmopteridae) off Juan Fernández Archipelago, Chile. Revista Biología Marina y Oceanografia, 45, 359-363.

54. Rodríguez SM, D'Elía G, George-Nascimento M. 2012. New host and geographical record for Mooleptus rabuka (Nematoda: Gnathostomatidae) in the largenose catshark Apristurus nasutus (Carchariniformes: Scyliorhinidae) off Juan Fernández Archipelago, Chile. Revista Biología Marina y Oceanografía, 47, 161-165.

55. Rubec LA, Hogans WE. 1988. Albionella fabricii n. sp. (Copepoda: Lernaeopodidae) from the gills of Centroscyllium fabricii from the Northwest Atlantic. Systematic Parasitology, 11, 219-225.

56. Sasal P, Mouillot D, Fichez R, Chifflet S, Kulbicki M. 2007. The use of fish parasites as biological indicators of anthropogenic influences in coral-reef lagoons: A case study of Apogonidae parasites in New-Caledonia. Marine Pollution Bulletin, 54, 1697-1706.

57. Vaughan DB, Chisholm LA. 2011. Amendment of Pseudoleptobothrium Young, 1967 (Monogenea, Microbothriidae) with the description of Pseudoleptobothrium christisoni sp. nov. from the dermal denticles of Rhinobatos annulatus (Rhinobatidae) off the southern tip of Africa. Acta Parasitologica, 56, 280-289.

58. Weigmann S. 2016. Annotated checklist of the living sharks, batoids and chimaeras (Chondrichthyes) of the world, with a focus on biogeographical diversity. Journal of Fish Biology, 88, 837-1037.

59. Williams HH, MacKenzie K, McCarthy AM. 1992. Parasites as biological indicators of the population biology, migrations, diet, and phylogenetics of fish. Reviews in Fish Biology and Fisheries, 2, 144-176.

60. Yano K, Musick JA. 2000. The effect of the mesoparasitic barnacle Anelasma on the development of reproductive organs of deep-sea squaloid sharks, Centroscyllium and Etmopterus. Environmental Biology of Fishes, 59, 329-339.

Cite this article as: Espínola-Novelo JF, Escribano R \& Oliva ME. 2018. Metazoan parasite communities of two deep-sea elasmobranchs: the southern lanternshark, Etmopterus granulosus, and the largenose catshark, Apristurus nasutus, in the Southeastern Pacific Ocean. Parasite 25, 53. 
Reviews, articles and short notes may be submitted. Fields include, but are not limited to: general, medical and veterinary parasitology; morphology, including ultrastructure; parasite systematics, including entomology, acarology, helminthology and protistology, and molecular analyses; molecular biology and biochemistry; immunology of parasitic diseases; host-parasite relationships; ecology and life history of parasites; epidemiology; therapeutics; new diagnostic tools.

All papers in Parasite are published in English. Manuscripts should have a broad interest and must not have been published or submitted elsewhere. No limit is imposed on the length of manuscripts.

Parasite (open-access) continues Parasite (print and online editions, 1994-2012) and Annales de Parasitologie Humaine et Comparée (1923-1993) and is the official journal of the Société Française de Parasitologie. 